Purdue University

C00-1428-38g

Contract No. AT $(11-1)-1428$

$$
\begin{aligned}
& \text { Annual Progress Report, Task B } \\
& \text { for June 1, } 1973 \text { to May 31, } 1974
\end{aligned}
$$

This $1 \mathrm{~g}$ the annual report by the high-energy theoretists in the physics departaent, Purdue University. This report consists of a list of personnel who have worked on this contract and the annual progress reports prepared by the Individuals.

\title{
I. List of Personne1:
}
R. H. Capps
(Professor)
S. P. Rosen
(Professor)
M. Sugawara
(Professor)
A. Tubis
(Professor)
L, A, P, Belázs
(Associate Professor)
N. H. Fuchs
(Asseciate Professor)
T. K. Kuo
(Associate Professor)
E. Fischbach
(Ass1stant Professor)
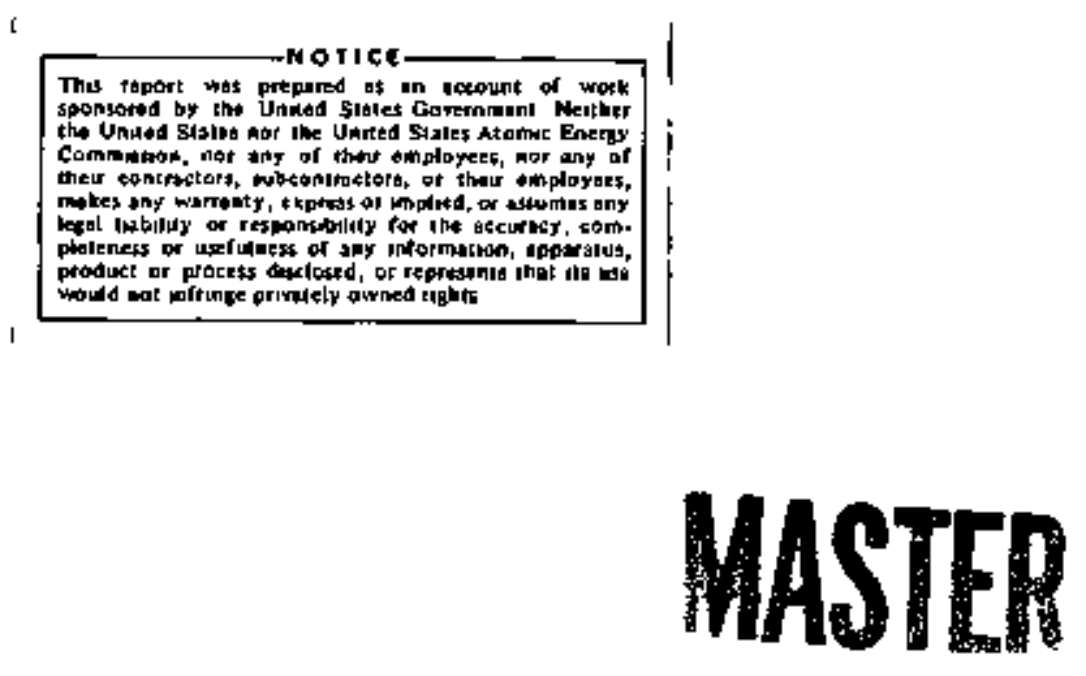


\section{DISCLAIMER}

This report was prepared as an account of work sponsored by an agency of the United States Government. Neither the United States Government nor any agency Thereot, nor any of their employees, makes any warranty, express or implied, or assumes any legal liability or responsibility for the accuracy, completeness, or usefulness of any information, apparatus, product, or process disclosed, or represents that its use would not infringe privately owned rights. Reference herein to any specific commercial product, process, or service by trade name, trademark, manufacturer, or otherwise does not necessarily constitute or imply its endorsement, recommendation, or favoring by the United States Government or any agency thereof. The views and opinions of authors expressed herein do not necessarlly state or reflect those of the United States Government or any agency thereof. 


\section{DISCLAIMER}

Portions of this document may be illegible in electronic image products. Images are produced from the best available original document. 
Purdue University

Contract No. AT(11-1)-1428

\section{Progress Reports:}

1. …‥ Caps:

Paper 1 on the accompanying list is concerned with the spplication of duality conditions to meson-baryon scattering amplitudes. It is pointed out that while varlous authors have used slightly different conditions, there are in general three types of solutions for the spectrum of baryon resonances. All of these accomodate the syometry group $\operatorname{SU}(6) \otimes 0(3)$. Two of these general solutions are in approxima te agreement with present experimental evidence; both give predictions expressable in terms of quarks. In one the spectrun Is that of the quark model with harmonic-oacillator forces. In the other, the resonances of opposite parities correspond to states of opposite synmetries in the exchange of two of the quarks in the baryon wave function. The tain tesults are the identffication of experimental phenomena that may best show which, if either, of the two solutions is approximately correct. These phenowena are the posstble existence of certain higher resonance multiplets, and some of the decay branching ratios of higher resonances.

Paper 4, written in collaboration with the graduate student Bruno Mathis, is concerned with Odorico's hypothesis that the zeros in hadron-hadron scattering amplftudes are linear functions of Mandelstam's energy and momentuntransfer variables. Odorico has pointed out that when this hypothesis is combined with certain experimental data fnvolving meson-baryon scattering, a violation of SU(3) Interaction symetry is inplied. However, the data analyzed by Odorico does not in ttself require su(3) violation. In paper 4 the origin of this conflict is explained. It is shown that Su(3) symmetry must be 
violated in the mesonic channel as well as the baryonic channels, in contradiction to one of Odprico's conclusions. Published experimental data on $\overline{\mathrm{K}} \mathrm{N} \rightarrow \pi \Sigma$ and $\pi \mathrm{N} \rightarrow \mathrm{K} \Sigma$ amplitudes are analyzed In an atcempt to resolve the conflict experimentally. The aralysis shows that the linear-zero hypothesis is better satisfied than SU(3) Interaction symmetry, although the results are not conclusive.

In paper 2 it is pointed out that the 1420 MeV $E$ meson cannot be a pure su(3) singlet. This is puzzing because other members of a possible Su(3) octet Including the $B$ have not been observed in the same mass range. The observed spin and paricy of the $\mathrm{E}$ are exther $0^{-}$or $1^{+}$. In a previous paper, I had shown that application of Odorlco's linear-zero hypothesis to meson-meson scattering leads to the prediction that the basic pseudoscalar meson nultiplet (that of the pion) Includes 10 members, seven of them octet members, one a singlet, and two singlet-octet mixtures. It is suggested that the $\mathrm{E}$ is one of these singlet-octet mixtures. This leads to a predicted partial width for the $\left(\mathrm{K}^{*}{ }^{*}+\overline{\mathrm{K}}^{*}\right)$ decay in agreement with experiment.

Papers 3 and 5 are derivations of properties of the Han-Nambu, threetriplet quark model. The symetry group of this model is su(3) su(3); each quark has two Indices, one referring to each of the SU(3) groups. One SU(3) is the observed group of Isospin and hypercharge, and the other. su(3) may be called the "color" group. The strongest forces th the model result from the exchange of an octet of vector mesoss coupled to the color ind 1ces . 
Purdue University

Contract No. AT(11-1)-142B

Paper 3 is an extension of some results of kambu and of Lipkin concerning the saturation properties of this nodel. Nambu had shown that the lightest states are predicted to be color singlets, and that a heavier bound state of two quarks (a "diquark") is also predicted. Lipkin had presented plausibiliky arguments that a composite of two quarks and two aptiquarks in a color singlet state may decompose into two $q \bar{q}$ color singlets (nesons). In paper 3 it is shown that if Lipkin's arguments are accepted, the only bound states predicted are the meson, the baryon ( 999 ) and the diquark.

In paper 5, the model is extended to the symnetry group SU(n) $\otimes$ SU(n), and the possibtlity of hadronfc "molecules" 1,8 considered. These molecules are defined as bound atates of chree or more constituents (quarks and antiquarks) for whtch an overall attraction can exist only if peirs of ettracting constituents are relatively close together. It is shown that in hadronhadron, hadron-quark, and hadron-diquark composites, the molecular attraction $1 s$ a decreasing function of the color group parameter $n$, and is much less than in the analogous cases of charged particles interacting by means of Coulomb forces. On the basis of this result, and similar results of other authors involving other kinds of forces, it is speculated that large' numbers of bound states of many hadrons do not exist in our universe only because the basic symretry group is fairly complicated.

\section{Papers Conpleted During Period, May 1 , 1973-Aprit 30, 1974}

1. "Duality and the Baryon Spectrum", Phys. Rev. Dㅁ, 3037 (1973).

2. "Possible Su(3) Classification of $\mathrm{z}$ Meson", Phys, Rev, Dg, 4202 (1973).

3. "Bound States in the Colored-Querk Model", Phys, Lett. 49. B, 178 (1974). 
Purdue University

$000-1428-389$

Contract No, AT $(11-1)-1428$

4. "Linear Zeros Versus SU(3) in Heson-Baryon Scattering" (with H. B. Machis), to be published in May 1, 1974 issue of Phys. Rev. D.

5. "An Effect of Internal Symetry on Hadronic BInding Forceg", (accepted by Phys. Rev. D.)

Io addition, the following papers, completed before the pertod, were published during the period.

1. "Exotic SU(3) Representatione in Inclusive Reactiong" (with H. B. Mathis), Phys. Rev, D7, 2734 (1973).

2. "Odorico's Bootstrap of Pseudoscalar Mesons", Phys, Rev. D7, 3394 (1973).

\section{S. P. Rogen:}

A. Nonlinear Lagrangiang and Chiral Symetry Breaking - (with H. Spivack):

We continued our work on nonlinear Lagrangians and chiral-symmetry breaking ${ }^{(1)}$ by studying the decay $\eta \rightarrow \pi^{+} \pi^{-} \pi^{\circ}$ using the pole model. We found that in order to account for both the observed rate and the observed slope for the decay to is necegsacy to brak the chiral symmetry (but not $\mathrm{SU}(3)$ ) in the kinetic part of the Lagranglan, and to Include two distinct representations (which. we take to be $\left(3,3^{*}\right) \oplus\left(3^{*}, 3\right)$ and the $(8,8)$ ) in the mass tern of the Lagrangian. The presence of these extra terms modifies our earlier predictions for $\pi^{-\pi}$ scattering lengths, the general effect being to increase then. (2)

Including symetry breaking terms in the kinetlc term of the Lagrangian is absolutely necessary in the concext of our model, but it has some other unwanted consequences. The algebta of charges derfved from the Lagrangisn, 
no" longer holds, and the space-time derivations of quantities no longer transform in the same way as the original quantities. (3) To avotd this difflculty ve will probably have to develop an entirely different model for $\pi \rightarrow 3 \pi$. Hoce that our qualtcative tesults are not affected by recent measurements of the $\pi$ width. (4)

For the tide being this work brings our effort on nonlinear Lagrangians to an end. There are sonte interesting problems in pion-nucleon scattering in which we can try and relate sun rules for scattering lengths to the sU(3) parameters of the symmetry-breaking lagrangian, but our tha jor research efforts are moving in other directions.

\section{B. Properties of Neucral Cycrents (In part wich $\mathrm{H}_{\text {. Spluack, and in part }}$} 1, th. F, Figchbach. B, Kayset, and G. Garuey):

The general philosophy of our work is that neutral currents are a new phenomenon, and we should therefore constder all possibitities for their propertes and devise as many experiments as we can to test them. The particular aspect upon which we focus our attention $1 \mathrm{~s}$ the poselbility that In the course of a neutral current interaction a neutrino can flip its spin. This imediately inplies that the current is, in fact, an adaixture of $S, T$, and $P$. We have proposed tests of this possibility in scatcering processes, in neutral beta decay and in pseudoscalar meson decay.

One particular calculation we are undertaking is the production of the $\mathrm{H}^{*}(3,3)$ resonance by neutral current interactions of neutrinos and nucleons. The ldea is to look for sons simple distribution such as $\dot{a}$ updown asymetry that is chsracteriatic of one-type of interaction $(e+8$. 
S, P, T) rather than another $(\mathrm{V}, \mathrm{A})$. This means that we must examine all possible Interactions and so the calculation is very involved.

\section{c. Wetmbers Angle as a Test of Gauge Theories:}

Georgi and Ghashos ${ }^{(6)}$ have recently proposed a unifled gauge theory of weak, electromagnetfc and strong interactions. They base it on the group SU(5), and the most imiediate consequence they obtain is that $\sin ^{2} \theta_{0}=3 / 8$. We have considered generalizations of this theory to the Broup SU(n) and have shown that $\sin ^{2} \theta_{w}=\frac{n-2}{2(n-1)}$. As a result $\sin ^{2} \theta_{w}$ must always be less than for these theorieg. Should the empirical value prove to be greater than $\frac{1}{2}$, the entire class of theorles can be eliminated.

\section{Weak Interact Ions and the Kalbfleigch-Powler Hodet of Leptons and Hadrons:}

Kalbfleisch and Fowler (7) have devised a model of leptons and hadrons which requires the existence of strange neutrinos. This in turn upsets the convent Lonal charged current description of Cabiblo universality between the moon, charged pion and charged Kaon lifetimes, by giving rise to new decay modes of the moon (manely $\mu-{ }^{-} \bar{\lambda}_{e} \lambda_{\mu}$ ). We have constructed a model which remains consistent with universality and which predicts that the ratio of strange to normal neutrinos in the final state of muon decay is $\tan ^{2} \theta_{c}$. However, we can no longer think of the cabibbo angle $\theta_{c}$ as a rotation angle in the SJ(3) of hadronic curtents.

1. S. P. Rosen and A. McDonald, Phys. Rev. D4, 1833 (1971); A. AcDonald and S. P. Rosen, Phys. Rev. D6, 654 (1972); A. McDona 1d, S. P. Rosen and T. K. Kuo Phys Letcera $\underline{40 B} 675$ (1972). 
Rurdue University

Contract No. AT $(11-1)-1428$

2. H. Splvack and S, P. Rosen, Phys, Rev, D8, 2877 (1973).

3. A.J. Macfarlane and P.H, Heisz, Nuovo Dimento Letters $\underline{2}, 25$ (1969).

4. A, Browan et a1, Phys, Rev, Letter 32, 1067 (1974).

5. B. Fischbach, B. Kayser, G. Garvey, and S. P. Rosen (report to London . Conference).

6. H, Georgi and S, L. Glashow, Phys. Rev. Letters 32, 438 (1974).

7. G. Kalbfleisch and E. Fowler, Nuovo Cinento 19A, 173 (1974).

\section{Paperg Published:}

1. A. McDonald and S. P. Rosen, Phys. Rev. D7, 3686 (1973).

2. A. MeDonald and S. P. Rosen, 3. Math. Phys, 14, 1006 (1973).

3. H. Spluack and S. P. Rosen, Phys, Rev, D8, 2877 (1974).

\section{In Press:}

'Weak Interactions and the Kalbfletsch-Fowler Mode1", S. P. Rosen, Nuovo Gimento 20A, 288 (1974).

\section{M. Sugawara:}

\section{Foss1ble Origtn of Symetry Bregking:}

If the Weinbers theory ${ }^{1}$ of weak interactions is normalizable primarily because of its gauge invartance, the hadronic Jagrangian must also be gauge Lnvariant alnce hadrons also partlcipate In weak interactions. As was often eruphasized by Weinberg ${ }^{2}$, however, this becomes a severe constraint on strong Interactions, especially if the basic hadrons are the pseudosclar mesons and spin- $\frac{1}{2}$ baryons. In thia case, the hadronic Lagrangian is written aside from mass terms, etc. As 


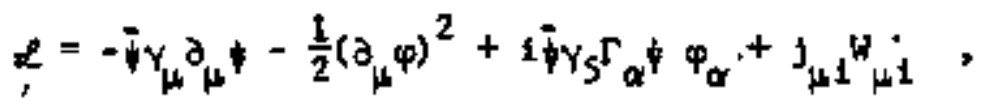

where $\Phi_{\alpha}$ is a component of $\psi, \Gamma_{\alpha}$ is Hermitian, and the hadronic weak current is

$$
j_{\mu 1}=i g \bar{\psi} Y_{\mu}\left(v_{i}+Y_{5} A_{1}\right)+i g \partial_{\mu} \varphi \theta_{1} \varphi-\frac{z^{2}}{2} \varphi \theta_{1} \theta_{j} \varphi w_{\mu j} .
$$

The gauge invariance of (1) requires ${ }^{3}$ that not only $v_{1} \pm A_{1}$ and $\theta_{1}$ are Hornitian and obey the SU(2) algebra, $\theta_{1}$ belng pure imaginary in addition, but also

$$
\left\{A_{1}, \Gamma_{\alpha}\right\}=0,\left[v_{1}, \Gamma_{\alpha}\right]=\Gamma_{\beta}\left(\theta_{1}\right)_{\beta \alpha},
$$

It is this first condition in (3) that is difficult to satisfy, yet unavoidable unless the strong coupling in (1) is of the vector type, when this condition becomes $\left[A_{1}, r_{\alpha}\right]=0$. This 18 why neutral vector gluons are being considered seriously ${ }^{2}$.

The purpose of this project is to find the most general solution to the conditions (3) when all the pseudoscalar and vector mesons are considered. We require only that $\Gamma_{\alpha}$ satisfies charge-independence of strong interactions, and the charged $A_{ \pm}=A_{1} \pm{ } A_{2}$ have both strangeness-conserving $(\Delta S=0)$ and 8 trangeness-violating $(\Delta s+0)$ parts, while only the former arises in the neutral $V_{3}$ and $A_{3}$. If we consider kaon interactions, for example, the nost general charge-independent interactions of kaons with nine baryons are, in terms of the simplifled but obvious notation,

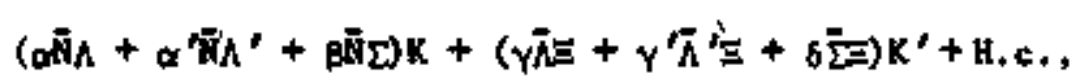

where $K$ and $K^{\prime}$ are $\left(K^{+}, K^{\circ}\right)$ and $\left(K^{0},-K^{+}\right)$, respectively. They can be 
written as, $\bar{\phi} \Gamma_{+} \psi K^{+}+\bar{W} \Gamma_{0} q K^{0}+H . c .$, where $\downarrow$ stands for $\left(P, N, A, A^{*}, \Sigma_{1}\right.$, $\left.\Sigma_{2}, \Sigma_{3},{\Xi^{0}}^{0} E^{-}\right)$. We thus study tf a $9 \times 9$ Heraltian matrix $A_{1}$ which has both $\Delta S=0$ and $\Delta S \neq 0$ parts can anciconmute with these $\Gamma_{+}$and $\Gamma_{0}$, by examining ats the matrix-elements of their anticomentators explicitly. We find 4 that such a matrix $A_{1}$ exiata only if

$$
\alpha^{2}+\alpha^{\prime 2}=\beta^{2}, \gamma^{2}+\gamma^{\prime 2}+2 \beta^{2}, \alpha \gamma+\alpha^{\prime} \gamma^{\prime}=0, \delta=0
$$

or $\left(\alpha, \alpha^{\prime}, \beta\right)$ and $\left(\gamma, \gamma^{\prime}, 6\right)$ are interchanged, where we have made all these coupling constant real by adjusting the fields in (4) stnce this is possible becauge of the last two conditions in (5). The conditions (5) leave only the overall constant and the ofixing angle undetermined. If the latter is defined thy $\alpha=\beta \sin \varphi, \alpha^{\prime}=\beta \cos \varphi, \gamma=-\sqrt{2} \beta \cos \varphi$, and $\gamma^{*}=\sqrt{2} \beta$ sinp, the kaon Interactions (4) and (5) can be written as

$$
1 f \sum_{\alpha=4}^{\gamma} \quad \bar{\psi}_{5}\left(F_{\alpha}-\dot{D}_{\alpha}\right) \psi \varphi_{\alpha},
$$

where $F_{\alpha}$ and $D_{\alpha}$ are the usuel $9 \times 9$ V(3) matrices,

$$
\phi_{g}=\Lambda \cos \varphi^{\prime}+\Lambda^{\prime} \sin \varphi^{\prime}, \phi_{0}=-\Lambda \sin \varphi^{\prime}+\Lambda^{\prime} \cos \varphi^{\prime},
$$

and $\varphi^{\prime}=\varphi_{0}-\varphi$, with $\varphi_{0}=35^{\circ}$ or Bin $\varphi_{0}=\frac{1}{\sqrt{3}}$. We also find ${ }^{4}$ that nooe of the charge-independent interactions of plons and $\eta$, $\eta^{\prime}$, with these baryons fo allowed in the atrong coupling In (1), because the corresponding $\Gamma^{\prime} \theta$ cannot antigomate with the above, matrix $A_{i}$. Thus, the koon coupling in (1) Is the one. Implied by the U(3) symmetry, but this U(3) synitotry 18 
broken by kaons since other mesons are excluded in (1). Here, the essentfal point is that this symetry breaking is related by gauge invariance to partiy violation in weak interactions.

We can solve the second condition in (3) in essentally the same way. The requirements that $V_{1} \pm A_{1}$ and $\theta_{1}$ obey the su(2) algebra and that the neutral $v_{3}$ and $\Lambda_{3}$ bave no $\Delta^{S} \neq 0$ part deteraine all $v_{1}, A_{1}$, and $\theta_{1}$, and thus the hadronic weak current, more ar less completely. We have considered ${ }^{4}$ a11 the charge-independent interactions of pseudoscalar and vector mesons with splo- $-\frac{1}{2}$ baryons. We have found ${ }^{4}$ few solutions, but only one appears to be consistent with the experinents. This only solution requires all nine baryons, but aljows orily kaons, to participate lo (1) as given by (6). In this solution, the charged weak current sacisfles all the selection rules of the Cabibbo current and time-reversal can be violated only ta the charged $\Delta \mathrm{S}=0$ vector current. All these are encouraging enough to wartant furthet studies on this project.

1. S. Heinberg, Phys. Rev. Letters 19, 1264 (1967); 27, 1688 (1971).

2. S. Weinberg, Phys. Rev. DB, 605 and 4482 (1973).

3. S, Heinberg, Phys, Rev, D2, 1068 (1973).

4. M. Sugawara, to be published.

\section{Arnold Tubis:}

A. Strong-, Electromagnetlc-, and Weak-Interaction Dynamics of Trinucleon Systens:

A major portion of my research time during the past year has been taken up with the preparation (In collaboration with Professor Y. B. K1m) 
Purdue University

$600-1428-389$

Contract Mo. AT (11-1)-1428

of a review article on recent developments in the theory of three-nucleon systems. The article was completed in February, 1974 and will be published in Yol, 24 of Annual Review of Nuclear Sclence (1974). I also pre* pared and delivered a sertes of lectures ${ }^{2}$ on the Faddeev theory of crinucleon bound states at the Finnish Sumer School in Nuclear Physics, Liperi, Finland, June 11-16, 1973. At present, Professor Y. E. Kim and I are completing a book manuscript on the three-nucleon problem for the Clarendon Press. My research on specific areas of the three-nucleon problem during the past year are detafled below tn separate subsections.

\section{a. S1ngular Core-Interaction and One-Plon-Exchange Constrsints on the_off-} Energy-Sheil Two-kucleon T-Matrix (with Y, E. Kin)

Baranger et al. (BGMS) (Nucl. Phys. Al38, 1 (1969)) have shown how to construct a partial-wave off-energy-she11 two-nucleon T-natrix, whose onshe11 (phase-shift) behavior is spectfied, without explicitly using an underlying potential. They show in particular that the full off-she11 T-matrix may be generated from an assuned form for the symmetric part o(p|k) of the half-off-shel1 I-matrix $\varphi(k \mid p)$, where $\varphi(k \mid k)=\frac{-2 k}{\pi} \sin \delta(k), \delta(k)$ being the phase shift. This construction should prove very useful in investigations of the sensitivity of the properties of few-nucleon systems to the off-shell two-nucleon T-matrix. The BGMS construction does not incorporate the congtraint assaclated with the firmly established one-pion-exchange (OPE) tail of the two-nucleon potentlal. It 18 alsa not applicable to two-nucleon interactions whose ghort-range behaviors are described by the boundary condition modeI (BCM) (H. Feshbach, E. L. Lomon, A. Tub1s, Phys, Rev. Lett. $\underline{6}$, 635 (1961)). Since there are considerable theoretical grounds for assuning 
that the effective two-nucleon core-interaction at low energies is well described by the BCM; we have generalized the BGMS construction so as to incorporate the constratots due to singular core interactions (described by the $\mathrm{BCM}$ ) as well as the OPE tail of the underlying two-nucleon interaction.

b. 3 He Rroperties from a Complete Solution of the Faddeev Equations with the Reid Soft-Core Potential (with $Y$, E. Kin and R. A. Brandenburg) ${ }^{4}, 5$ :

The ${ }^{3} \mathrm{He}$ binding energy $\mathrm{E}_{\mathrm{B}}$, wave function, and ellectronagnetic form faccors are calculated from a complete solution of the Faddeev equations with the full Reld soft-core potential (RSCP). We find $E_{B}-6.9 \mathrm{HeV}, P(S)=$ $89.5 \mathrm{~B}^{2}, \mathrm{P}\left(\mathrm{S}^{\prime}\right)=1.60 \%, \mathrm{P}(\mathrm{D})=8.77 \%$, and $\mathrm{P}(\mathrm{P})=0.05 \%$ in good agreement with the results of a previous calculation using only the ${ }^{1} \mathrm{~S}_{0},{ }^{3} \mathrm{~S}_{1}-{ }^{3} \mathrm{D}_{1}$ components of the RSCP (E. P. Harper, Y. E. KIm, A. Tubis, Phys. Rev, Lett, 28, $1533(1972)$ ). Our results suggest that the use of on1y ${ }^{1} s_{0},{ }^{3} s_{1}-{ }^{3} g_{1}$ components of the RSCP glves relative errors of $₹ 2.5 \%$ in calculated trinucleon binding energies and wave-function probabilitles.

The calculated ${ }^{3} \mathrm{He}$ electromagnetfc form factors are in good agreement W1th experimental data for monentum transfer squared $Q^{2} \approx 10 \mathrm{~F}^{-2}$ but do not give a good fit for $Q^{2}>10 \mathrm{~F}^{-2}$ gven after relativistic corrections are added. Previous calculations (E. P. Harper, Y, E. Xim, A. Tubis, Phys. Rev. C6, 1601 (1972)) Indicate that non-1ocal phase-equivalent modifications of the RSCP (which are compatible with the deuteron properties and leave the potential essentially unchanged for $r \$ 1 F$ ). do not substantialty reduce the discrepancy with experdment of the calculated $\mathrm{E}_{\mathrm{B}}$ and electrouganetic form factora. This by taken as strong evidence for three-nuclean forkes and exchange-current contributions to the form factors. 
Purdue University

c. Medium-Energy Proton Radlative Capture by Deuterons (with B. Craver and Y. E. Kim):

The radiative capture reaction ${ }^{2} H(P, \gamma){ }^{3}$ He at intermediate energies has been calculated using the ${ }^{3}$ He wave function discussed in reference 4 . Preliminary resulto Indicate that substantial contributions from mesonexchange currents will be required to fit the experinentil data.

B. Ugper and Lower Bounds on High Energy Cross Sectlons (with_B, Craver)

We have derived upper and lower bounds for weighted integrals of $2 \sigma_{s}=$ $\sigma_{\text {cot }}\left(\pi^{+} p\right)+\sigma_{\text {tot }}\left(\pi^{-} p\right)$ at pion laboratory energies $\omega>60 \mathrm{GeV}$ in terms of avallable experimental data and assumed bounds on the phase of $T_{g}(w)$, the $\pi p$ crossing-symetcy forward scattering amplitude above $20 \mathrm{GeV}$. The bounded integrals are of the form $\int_{s}^{M} \sigma_{s}(\omega) K(w)$ dw with $60 \mathrm{GeV}<M_{1}<M$, and $K(w) \sim w^{-\gamma}(1<\gamma<1,72)$ as $w \rightarrow \infty$, Our analysts also gives an upper bound on $\delta_{1}$, the minimum of the phase of $T_{s}(w>60 \mathrm{GeV})$. These bounds are more sensitive to the very-bigh cross-section behavior than are previously derived bounds (T,N. Pham, T. N. Trong, Phys, Rẹv, Lett. 31, 330 (1973)). Along with these other bounds they may be used directly to test the consistency of $\pi^{ \pm}$data with forward dispersion relactons and to constrain the parameters in theoretical models of high energy scattering amplitudes.

1. Y. E. Kin, A. Tubis, "The Theory of Three-Nucleon Systems," to be pub-" 11shed in Vol. 24 of Annugl Review of Nuclear Science (1974).

2. A. Tubis, "Selected Topics in the Theory of Trinucleon Bound States," lectures given at the Finnish Sumarer School in Nuclear Physics, 
Liper 1, Finland, June 11-16, 1973.

3. Y. E. K1m, A. Tubis, "Singular-Core-Interaction and One-Pion-Exchange Constraints on the Off-She1t Two-Nucleon T-Matrix," Pbys, Rev, Letc. $31,952(1973)$

4. R, A, Brandenburg, Y, E. Xim, A. Tubis, "Trinucleon Properties from a Complete Solution of the Faddeev Equations with the Reid Soft-Core Potent1a 1," Phys, Lett, B49, 205 (1974).

5. R. A, Brandenburg, Y, E. KIm, A. Tub1s, "Magnetic Formi Factor of "He," submitted to Phys. Rey, Lett.

6. B. Craver, A. Tubls, "Upper and Lower Bounds for the Sum of $\pi^{+} p$ and $\pi$-p Cross Sections at High Energy," submitted to Phys. Rey. D.

5. L. A. P. Balázs: Most of the work during the preceding year has been on applications of f/P universality to inclusive processes in the triple-Regge region. This region is Important because in principle it permits us to directiy extract triple-Regge couplings. These couplings are the basic input tato many dynamical calculations of high-energy processes. Unfortunately, a large number of terms are needed to describe the data in our region. Even after we impose exchange-degeneracy wo require st lessot the PPP, PPR, RRP and RRR terms, where $P$ is the Pomeron and $R$ the leading meson trajectory. The number of Independent couplings can be reduced considerably, however, if we have some kind of syametry or universality schene which would relatè the $R$ and $P$ to each other. 
Several years ago, Ghew and Snider ${ }^{\prime}$ and Carlitz, Greet and zee ${ }^{2}$ proposed that the f-P Regge-residue ratio $r$ is a universal quanticy, independent of the process involved, which could be either a physical process or Regge-particle "scattering"" This permits us to first extract $r$ from a two-body process $1 \mathrm{kke} \mathrm{PP} \rightarrow \mathrm{pp}$ and then use 1t to relate, say, iif to ilf $(1=P, R, \ldots)$ : We can then immediately predict the deviation from scalIng of $\mathrm{pp} \rightarrow \mathrm{pX}$ in terms of $\mathrm{r}^{4}$ The resuits are found to be in good agreement with the recent data from NaL. 5,6

In addition to the above relations, we can also use $f / P$ universality to relate PPi to $\mathrm{ffi}$ and $\mathrm{fP}$, again in terms of $r$. Exchange degeneracy then enables us to relate the last two to $\mathrm{RI}$ and $\mathrm{RP}_{2}^{\mathrm{V}}$. The upshot is that we can describe all the couplings for a process like $\mathrm{pp} \rightarrow \mathrm{pX}$ in terms of only a single new parameter, say the PPP coupling (n exchange has to be treated separately but one can write nodels for ft which do not involve any new parameters). We can therefore predict all the shapes of the distributions in the tulsaing masa $M^{7}$ The results are again in fairly good agreement with the recent Mat data. ${ }^{6}$

Our scheme is particularly useful when inclusive data has been incegrated over the missing mass, as in the small-mouentum-transfer (t) data of Childress et a $1 .^{B}$ Work has been started on using our scheme to extract the PPP coupling from this daca at very smalt $t$. In this way we hope to see whether this coupling shows any cendency to vandsh at $t=0$, as predicted in cercain theories. Our scheme can also be combined with factorization to derive relations between different processes, even in a non-scaling 
reg1on. For example pp $+\mathrm{pX}$ can be related to $p^{-}+\mathrm{pX}$. Work has been begun to test such a relation, with encouraging preliminary results. Finally, our schene permits us to use finite-nass sun rules to predict tripleRegge couplings in terns of low missing-mass daca, which is easier to measure experimentally. Rreliminary calculations seem to ind cate that these sum rules are in fact satisfied.

In addition to the above program, work was conpleted on cercain app1ications of "nonlinear" duality to mir scattering. In this type of duality the low and intermediate-energy absorptive part in states and energy regions which do not contain any narrow, resanances are built up'via unitartey in terms of quas 1-two-body amplitudes which are thenselves assumed to be we11 approximated by Rigge exchange, even at relatively low energles. In states and energy regions which do contain narrow resonances, these are put in by hand; ordinaçy linear duality may be used to obtain thelr widths, Once we know the low or internediate-energy absorptive part we can calculate highenergy Regge parameters via fintte-energy sun rules. Previously this approach had been used to calculate average multiplicities at high energies 9 and to study the zero-structure of tensor and vector exchanges, which were found not to satis fy exchange-degeneracy. The latter calculation was then extended to an approxinate calculation (neglecting diffractive components) of the magaltude and width of the diffraction peak. 10,11 The results were found to be In reasomable agteement with experiment.

1. G. F. Chew and D. R. Snider, Phys, Rev, D3, 420 (1971). 2. R. Carl1tz, H. B. Green and A. Zee, Fhys, Rev. D4, 3439 (1971). 
Purdue University

Contract No. AT(11-1)-1428

3. L. A. P. Balazs, Phys. Letters 40, 269 (1972).

4. L. A. 9. Baláz, Phys. Letters 48B, 232 (1974).

5. F. Sannes et al., Phys. Rev. Letters 30, 766 (1973).

b. K. Abe at al., Phys. Rev. Letters 31, 1530 (1973).

7. L. A. P. Baläxs, A. Description of $p p \rightarrow p X$ in the Triple-Regge Region Incorporating f/P Untversality, Purdue Untversity preprint (1974).

8. S. Childress et a1,, Phys, Rev, Letters 32., 389 (1974).

9. L. A. P. Balăzs, Phys. Letters 45B, 40 (1973).

10. L. A. P. Balázs, Phys. Rev. D9, 231 (1974).

11. L. A. P. Balázs, Phys, Rev. D9, 237 (1974).

6. H. Fuchs:

\section{Broken SU(6) Symmatry:}

We have studied ${ }^{l}$ the algebralc properties of the transformation $v$ between the constituent and current quark bases ${ }^{2}$ in the case when sU(3) is broken for the current quatks. In a simple model, we showed that SU(4), gay sti11 be an exact symatry in the constituent quark basis but that sU(6) w can not. The forunlation was done in the context of currents integrated over timelike surfaces, which is the traditional way. However, In order to discuss collinear symetry such as $\mathrm{SU}(6)_{W}$, without any problems of taking infinite momentum 1 intes, th is better to define all operators and comulators on a lightlike surface. Then the transformation $V$ from current to constituent quark basis is not unique even to the free symetric quark nodel until further angular constraints are Imposed in order to ensure that the states con* nected by SU(6) operators have the correct spin properties. On the free 
quark mode1, but with quark mass splitting, we show ${ }^{3}$ that no such transfarmation exists. It is possible, howeve $x$, to satisfy all the constraint equations which only tnvolve strangeness preserving operators. The transformation $V$ is then essentially unique. We investigaced the algebraic properties of $V$, finding then more complicated than that of $v$ of the sU(3) symetric mode1. We further attempted to obtain sone results for a model with interaction, but we could only quke litcle headway. Some hincs of the origin of mass breaking in SU(6) oultiplets did appear, however.

1. N. H. Fuchs, Phys. Rev. D8, 4079 (1974).

2. H. J. Welosh, Phys. Rev, D9, 1095 (1974).

3. H. H. Fuchs, prepriat (1974); submitted for publication.

7. I. K. Ku:

\section{su(4) $\times$ su(4) symetry:}

Based on lepton-hadron analogy, SU(4) $x$ SU(4) was suggested as the symmetry group of the leptons as well as the hadrons. This problem was studied further, both by anself and by others.

For the weak and strong interactions, a renotmalizable gauge theory based on SU(4) $x$ SU(4) was constructed ${ }^{2}$. From the trong interaction point of view, the $(4, \overline{4})+(\overline{4}, 4)$ type symetry breaking was analyzed by several authors ${ }^{3}$. Their approach is rather interesting. However, the numerous epproxinstons necessary in the analysis do not lend credence to their final numerical results. Inproved analysts would certainly be welcone. We have amalyzed $\operatorname{su}(4) \times \operatorname{su}(4)$ in another way ${ }^{4}$. It is pointed out 
that there exists a finite SU(4) $x$ SU(4) rotation which acts as a mass reversal operator for the quarks. Specifically, we define

$$
\widetilde{W}=\exp 1\left(4_{\pi} z_{-}\right)=\tilde{W}^{-1} \text {. }
$$

where 2 . is the $15^{\text {th }}$ generator of SU(4) - as defined in Ref. 4. We then have

$$
\begin{aligned}
& \tilde{w}(\bar{q} q) \tilde{w}=-\bar{q} q, \\
& \tilde{\omega}\left(\bar{q} \gamma_{\mu} \partial_{\mu} q\right) \tilde{w}=+\bar{q}_{\gamma_{\mu} \partial_{\mu} q} .
\end{aligned}
$$

1

where $q$ is the quark field operator. It is also easy to see that

$$
\text { นัp }=(-1)^{42} \mathrm{P} \text {, }
$$

and that $\tilde{W}$ comoutes with the whole $\operatorname{sU}(4) \times \mathrm{SU}(4)$ group.

The physical meaning of $\tilde{\mathbf{w}}$ is that it changes the parity of all the charmed states relative to the uncharmed ones. Since this relative parity is physically not measureable, $\tilde{W}$ is a syquetry operator. F leaves the kinetic energy term In a Lagranglan Eield theocy lnvariant, but changes the sign of the quark ness term, It follows that the bare quark masses must vanish. This result is suggestive of the parton picture, in which the partons are veny 1 ight constituents of the hadrons. On the other hand, the effective quark mases may be non-vantahiag. Indeed, if the symmetry is spontaneously broken, scalar mesons could give rise to quark nasses. It is painted out that this is very likely the situstion in reality. It suggests that the SU(4) $x$ SU(4) symotry is broken in two stages. The symetry is first broken spontaneously, with the vacuun invariant only under Su(3). Here the charmed quark pleks up its mass. In the second stage, the residual su(3) $x \mathrm{Su}(3)$ 
symatry is broken, this time both explicitly and spontaneously.

1. P. Dittnęr, S. Eliezer and T. K. Kuo, Phys. Rev. Lett. 30, 1274 (1973).

2. D. Ross and S, Eliezer, (to be publlshed); D. Ross, (to be published).

3. D. Dicus and V. S. Whur, Fhys. Rev. DQ, 1003 (1974); Rhys. Lett.

49B, 171 (1974). S. C. Prasad, Phys. Rev. D9, 1017 (1974).

4. T. K. Ku10, Phys. Rev., to be published.

\section{B. E. Fischbach:}

\section{Phenouenology of Meson Interactions in the DKP-Foxnal 1sm:}

As detailed in our previous proposal we have continued our program of exploring the description of meson Interactians based on the Dufin-KennerPetiau (DKP) equation. This past year has seen the publication of three additional papers in this sequence. $1,2,3$ In the first of these, the previously discussed comparison of the Klein-Gordon (KG) and DKP descriptions of the PBB coupling constants was given. It was shown that the $x^{2}$ for the DKP fit to the $\pi N N, \pi A \Sigma, \pi \Sigma \Sigma$, KNA and KNE coupling congtants was only 0.25 compared to $x^{2}=8.9$ for the $K G$ f1t. Sone subsequent analyses supported these results by noting that the predtcted value of $\theta=0 / D+F=0.71 \pm$ 0.07 from the DKP fit suggested that the TNN coupling should be very small, stace it would vanish exactly for $\beta=0.75$. Preliminary analysis of the experimental data suggest that the TNN coupling constant is indeed small. In two relaced papers ${ }^{2,3}$ the mathematical structure of the DKP algebta was analyzed by déconposing the DKP algebrs Into spin projection operators, for $J \cdot 0$ and $\mathrm{J}=1$. The purpose of this decomposition was to facilitate 
the application of the DKP formalism to actual physical problems involving particles of spin-0 or 1 . Some applications of the general formalisn were given including a proof that the KG and DKP meson currents are not equivalent in the presence of SU(3) symmetry breaking.

1. F. T. Meiere, E. Fischbach, A. McDonald, M. M. Nieto, and C. K. Scott, Phys. Rev. Dis, 4209 (1973).

2. E. Fischbach, M. Y4. Mieto, and C. K. Scott, J. Math. Phys. 14, 1760 (1973).

3. E. Fischbsch, J. D. Louck, M. M. Nieto, and C. K. Scott, J. Math. Phys. 15, $60(1974)$. 